Elke Rohmann, Beate Küpper und Martina Schmohr

\title{
Wie stabil sind Bindungsangst und Bindungsvermeidung? Der Einfluss von Persönlichkeit und Beziehungsveränderungen auf die partnerbezogenen Bindungsdimensionen
}

How stable are attachment anxiety and attachment-avoidance? The influence of personality and changes of relationship on partner-related attachment dimensions

\begin{abstract}
Zusammenfassung
Es wurde in einer Studie mit einer Stichprobe von 57 Studierenden, die zu drei Messzeitpunkten innerhalb von acht Monaten befragt worden waren, untersucht, ob Persönlichkeitsmerkmale (Verträglichkeit und Neurotizismus) besser die allgemeine Bindung als die partnerbezogene Bindung vorhersagen. Diese Annahme konnten wir für die Vorhersage von Bindungsangst durch Neurotizismus bestätigen. Weiterhin ließ sich in einer cross-lagged-panel-Analyse zeigen, dass die partnerbezogene Bindungsangst nach acht Monaten besser durch Neurotizismus vorhergesagt wurde als umgekehrt. Dagegen ließ sich für den Zusammenhang von Verträglichkeit und partnerbezogener Bindungsvermeidung keine einseitige Einflussrichtung feststellen. Die Veränderbarkeit der partnerbezogenen Bindungsangst konnte durch positive und negative Beziehungsveränderungen vorhergesagt werden. Nach negativen Beziehungsveränderungen nahm die Bindungsangst zu, während sie nach positiven Beziehungsveränderungen abnahm. Entgegen unserer Annahmen fanden wir keine geringere Stabilität von partnerbezogener Bindungsangst im Vergleich zu partnerbezogener Bindungs-
\end{abstract}

vermeidung. Zudem waren die beiden partnerbezogenen Bindungsdimensionen zeitlich genauso stabil wie Verträglichkeit und Neurotizismus.

Schagworte: Partnerbezogene Bindung, allgemeine Bindung, Neurotizismus, Verträglichkeit, zeitliche Stabilität, Beziehungsveränderungen

\section{Abstract}

In a sample of 57 students who were interviewed at three waves within eight months the assumption was investigated that personality dimensions (specifically agreeableness and neuroticism) predict general attachment better than partner-related attachment. In accordance with this assumption neuroticism predicted general attachment anxiety better than partner-related anxiety. Results of a cross-lagged panel analysis showed that partner-related anxiety was predicted better by neuroticism than vice versa. With respect to the association between agreeableness and partner-related avoidance, no dominant influence of one variable over the other was detected. After positive and negative changes in the course of their relationship congruent changes in 
partner-related attachment anxiety were detected. Specifically, negative changes in the course of relationship caused an increase in attachment anxiety, whereas positive changes in the course of relationship caused a decrease in attachment anxiety. Contrary to our expectations, the stability of partnerrelated attachment anxiety did not differ from the stability of partner-related attach- ment avoidance. In addition, both dimensions of partner-related attachment reached the same level of temporal stability as agreeableness and neuroticism.

Key words: partner-related attachment, general attachment, neuroticism, agreeableness, temporal stability, changes in relationship

\section{$1 \quad$ Einleitung}

Bindung ist nach Maccoby (1980) als eine relativ dauerhafte emotionale Orientierung an eine andere Person zu verstehen. Das Konstrukt der partnerbezogenen Bindung, das die emotionale Orientierung von Erwachsenen in Liebesbeziehungen beschreibt, hat in den letzten Jahren zunehmend an Beachtung gewonnen. Theoretische Annahmen und die daraus abgeleiteten empirischen Untersuchungen (z.B. Hazan/Shaver 1987) hinsichtlich der Bindung an einen Partner beruhen auf der Bindungstheorie von Bowlby (1969/1986) und Ainsworth, Blehar, Waters und Wall (1978). Bowlby und Ainsworth et al. gehen davon aus, dass sich in der frühen Kindheit aufgrund der Erfahrungen mit den Bezugspersonen Annahmen über deren Verfügbarkeit bilden, die dann in internen Arbeitsmodellen kognitiv repräsentiert werden. Interne Arbeitmodelle sind als kognitive Strukturen oder Schemata zu verstehen, die Handlungspläne und Verhaltensweisen umfassen, die den Bedürfnissen nach Schutz, Sicherheit und Bindung dienen (Bierhoff 2000). Ainsworth et al. klassifizieren aufgrund ihrer Beobachtungen von ca. einjährigen Kindern in der „Fremden Situation“ einen sicheren, einen ängstlich-ambivalenten und einen vermeidenden Bindungsstil. Hazan und Shaver (1987) übertrugen das Konzept von Bowlby und Ainsworth et al. auf die Bindung an einen Partner und stellten durch Selbstklassifikation bei Erwachsenen ebenfalls einen sicheren, einen ängstlichen-ambivalenten und einen vermeidenden Bindungsstil an den Partner fest. Interindividuelle Differenzen zwischen Personen mit unterschiedlichen Bindungsstilen wurden seitdem eingehend untersucht, empirisch bestätigt (für einen Überblick siehe Shaver/Mikulincer 2002a) und mit anderen sinnverwandten Aspekten in der Partnerschaft, wie beispielsweise der Zufriedenheit in der Paarbeziehung, in Verbindung gebracht (u.a. Hazan/Shaver 1987). Die partnerbezogene Bindung hat sich in diesem Sinne als erfolgreiches Konstrukt erwiesen. Jedoch sind einige wesentliche Aspekte der Konzeption von Bindung bisher nicht zufriedenstellend geklärt worden. Dies gilt z.B. für die zeitliche Stabilität der partnerbezogenen Bindung. Die bisherige Forschung zusammenfassend, stellen Ross und Spinner (2001) fest, dass in Längsschnittstudien, die eine Zeitspanne von sechs Monaten bis zu vier Jahren umfassen, bei 20 bis 36\% der Stichprobe ein Wechsel des Bindungsstils nachweisbar ist. Die eindeutige Interpretation dieser Größe als „Veränderung der partnerbezogenen Bindung“ ist allerdings problematisch. Ein 
Grund sind die verschiedenen nebeneinander existierenden Erhebungsinstrumente. Die Vergleichbarkeit der mit ihnen erzielten Ergebnisse und die Reliabilität der Messungen sind teilweise fraglich. Neben diesen messmethodischen Problemen ist unklar, wie die ca. 30\% Flexibilität und 70\% Stabilität der partnerbezogenen Bindung letztlich zu beurteilen sind. Nach der Bindungstheorie stellen die veränderten Bindungsrepräsentationen eine Anpassung an (neue) Interaktionserfahrungen dar.

\section{Die partnerbezogene Bindung}

\subsection{Konzeption der partnerbezogenen Bindung}

Zwar sind die spezifischen Verhaltensweisen bei engen Bindungen von Kindern und Erwachsenen unterschiedlich, doch erfüllt die Bindung bei beiden eine sehr ähnliche Funktion (Shaver/Clark 1996). Auch in romantischen Beziehungen hat das Bindungsverhalten die Funktion, in bedrohlichen Situationen die Nähe des Partners herzustellen, sich Unterstützung zu sichern oder Angst zu reduzieren. Hazan und Zeifman (1999) zeigen, dass diese Sicherheit, die sich Beziehungspartner im Idealfall gegenseitig bieten, genau wie die Bindung zwischen Kind und Fürsorgeperson, einen adaptiven Vorteil darstellt.

In Übereinstimmung mit Collins und Read (1994) fassen wir die partnerbezogene Bindung als einen Teil der gesamten Bindungsrepräsentationen einer Person auf. Wir gehen davon aus, dass Personen gegenüber verschiedenen Zielpersonen und in verschiedenen Beziehungsformen unterschiedliche Bindungen aufweisen. Repräsentiert sind diese in mehreren, sich wechselseitig beeinflussenden Arbeitsmodellen, von denen angenommen wird, dass sie in Form eines hierarchischen Netzwerkes strukturiert sind. Nach Shaver, Collins und Clark (1996) sind an der Spitze dieser Hierarchie die generellsten Repräsentationen gespeichert, die auf Beziehungserfahrungen mit Eltern und anderen wichtigen Bezugspersonen zurückgehen. Diese abstrakten und generellen Modelle werden auf sehr viele Beziehungen und Situationen angewandt. Auf einem mittleren Abstraktionsniveau befinden sich dagegen spezifischere Arbeitsmodelle, die konkrete Beziehungsformen repräsentieren. Auf unterster Ebene sind die Modelle angeordnet, die der Bindung an einen spezifischen Partner entsprechen.

Diese Vorstellung eines hierarchischen Netzwerks erklärt einerseits die Beziehungsspezifität von Bindung, die sich in Studien, die verschiedene Beziehungsformen berücksichtigen, zeigt (Asendorpf/Banse/Wilpers/Neyer 1997; Mikula/Leitner 1998), ermöglicht aber andererseits auch die Annahme einer stark abstrahierten allgemeinen Bindung, die sich aus der Erfahrung mit zentralen Bindungsfiguren ableitet und einen Zusammenhang mit verschiedenen Arbeitsmodellen aufweist (Collins/Read 1990). Obwohl dies eine vorläufige Vorstellung ist, hat sie umfangreiche empirische Forschung zur Struktur und Organisation des Netzwerkes von Arbeitsmodellen hervorgerufen (z.B. Baldwin/Keelan/Fehr/Enns/KohRangarajoo 1996; Cozzarelli/Hoekstra/Bylsma 2000; Ross/Spinner 2001; Zim- 
mermann 1999). Die Konzeption eines hierarchischen Netzwerkes legt nahe, dass die partnerbezogene Bindung einerseits Zusammenhänge mit der allgemeinen Bindungsorientierung aufweist, andererseits aber auch durch spezifische Erfahrungen in Partnerschaften beeinflusst wird (Shaver/Mikulincer 2002b).

Sowohl theoretische Überlegungen (Bartholomew 1990) als auch empirische Befunde (Brennan/Clark/Shaver 1998; Grau 1999) begründen die Annahme von grundlegenden Bindungsdimensionen ,hinter“ den Bindungsstilen. Bartholomew benennt diese Dimensionen zunächst „Modell vom Selbst“ und „Modell von Anderen“, aber sie und ihre Mitarbeiter nutzen parallel dazu auch die Bezeichnungen „Angst“ und „Vermeidung“ (nach Brennan/Clark/Shaver 1998: 50). Zugrunde liegen dabei die Annahmen, dass ein negatives Modell vom Selbst eng verknüpft ist mit der Angst, verlassen zu werden und ein negatives Bild von Anderen stark mit vermeidendem Verhalten assoziiert ist. Mit den entsprechenden Fragebögen von Brennan, Clark und Shaver (1998) bzw. Grau (1999) lassen sich die Dimensionen Angst und Vermeidung in romantischen Beziehungen hoch reliabel erfassen.

\subsection{Die Veränderungen der partnerbezogenen Bindung}

Bowlby (1969/1986) nimmt an, dass die mentale Repräsentation der Bindung durch die inneren Arbeitsmodelle zwar eine gewisse Stabilität aufweist, aber trotzdem die Anpassungsfähigkeit des Individuums an die Umwelt gewährleisten soll. Bretherton und Munholland (1999) betonen, dass veränderte Bedingungen in der Interaktion zu veränderten Bindungsrepräsentationen führen.

Auch die partnerbezogene Bindung von Erwachsenen weist in empirischen Studien eine gewisse Veränderbarkeit auf. Die Ergebnisse einer Längsschnittstudie von Rohmann, Bierhoff, Schmohr und Ambrosy (1998) zeigen, dass die Sicherheit der Bindung an den aktuellen Partner bei Paaren, die in einer festen Partnerschaft (mittlere Beziehungsdauer = 8.2 Jahre) lebten, über einen Zeitraum von 14 Monaten abnahm.

Baldwin und Fehr (1995) schlagen vor, das wiederholt aufgetretene Ergebnis, dass sich bei ca. $30 \%$ der untersuchten Personen Veränderungen in der partnerbezogenen Bindung nachweisen lassen, nicht als Zeichen für ein unreliables Messinstrument zu werten, sondern als Teil des Konstrukts selbst zu verstehen. Collins und Read (1994) kommen in Bezug auf die inneren Arbeitsmodelle zu dem Schluss, dass diese sowohl Stabilität als auch Wandel durch Assimilation und Akkomodation zeigen.

Einerseits kann sich die partnerbezogene Bindung unter konstanten Beziehungskonstellationen als eine interpersonelle Disposition erweisen, die dann auf die Prozesse in romantischen Beziehungen einen Einfluss hat (Schmohr 2003). Andererseits kann die aktuelle partnerbezogene Bindung die zurückliegenden Beziehungserfahrungen reflektieren (Rohmann 2000), und damit veränderbar und spezifisch für eine bestimmte Beziehung sein (Feeney 1999). Cozzarelli, Karafa, Collins und Tagler (2003) fanden bei Frauen, die einen Schwangerschaftsabbruch vorgenommen hatten, einen mäßigen Zusammenhang zwischen dem vor und zwei Jahre nach dem Abbruch erfassten Bindungsstil (Barholomew/Horrowitz 1991) 
gegenüber emotional nahe stehenden Personen $($ Kappa $=.33$ ). Auch in der Studie von Lopez und Gormley (2002) zeigte sich nur eine moderate Stabilität des Bindungsstils (41\% bis $65 \%)$.

Da Bindungsvermeidung vorrangig durch das eigene Verhalten (z.B. Distanzierung) realisiert wird, sollte diese Dimension der Bindung stabiler sein als die Dimension Angst, die eher vom aktuellen Verhalten des Partners abhängig ist (Grau 1999). So findet Grau über einen Zeitraum von sechs Monaten für die zielpersonspezifische Bindungsvermeidung eine signifikant höhere Stabilität als für zielpersonspezifische Bindungsangst.

Bislang ist jedoch unklar, inwieweit besondere Lebens- oder Beziehungsereignisse bei Erwachsenen einen Einfluss auf die Stabilität des Bindungsstils haben. Davila, Karney und Bradbury (1999) identifizieren als Faktoren, die eine Veränderung der Bindungssicherheit im Laufe von zwei Jahren bei Jungverheirateten vorhersagen können, sowohl intra- als auch interpersonale Faktoren: Obwohl die Jungverheirateten im Laufe von zwei Jahren immer sicherer wurden, nahm die Bindungssicherheit beim Vorliegen von stabilen Vulnerabiltätsfaktoren (Störungen der Persönlichkeit, familiäre Psychopathologie, problematischer Familienstatus, wie z.B. Trennung oder Scheidung der Eltern) und ehelicher Unzufriedenheit ab. Trennungen scheinen hingegen auch zuvor sichere Personen unsicher werden zu lassen (Cozzarelli/Karafa/Collins/Tagler 2003; Kirkpatrick/Hazan 1994). Ruvolo, Fabin und Ruvolo (2001) finden, dass die Trennung vom Partner zu einer unsicheren Bindung bei Studentinnen führte, während diejenigen Studentinnen, die über einen Zeitraum von fünf Monaten mit ihrem Partner zusammen blieben, an Sicherheit dazu gewannen, was darauf hinweist, dass konkrete Beziehungserfahrungen einen Einfluss auf die partnerbezogene Bindung haben. So zeigt Küpper (2002), dass der aktuelle Beziehungsstatus (mit oder ohne feste Partnerschaft) mit der Bindung in Zusammenhang steht: Singles sind sowohl bindungsängstlicher (wenn sie unfreiwillig Single sind) als auch bindungsvermeidender (wenn sie freiwillig Single sind).

\subsection{Partnerbezogene Bindung und Persönlichkeit}

Persönlichkeit wird nach Neyer (2003) und Asendorpf (1999) als die Gesamtheit aller dauerhaften Eigenschaften (z.B. Temperamentsmerkmale, Einstellungen, Werthaltungen, Motive und Aspekte des Selbstkonzepts) definiert, die die Person von anderen Menschen unterscheidet. Die individuelle Besonderheit in Abgrenzung zu anderen Personen und die relative zeitliche Stabilität der Persönlichkeit sind hierbei zentral. Costa und McCrae (1985) beschreiben die Persönlichkeit in einem Fünf-Faktoren-Modell (Big Five) durch die Dimensionen Extraversion, Neurotizismus, Gewissenhaftigkeit, Verträglichkeit und Offenheit. Shaver und Brennan (1992) finden bedeutsame Zusammenhänge zwischen zwei dieser zentralen Persönlichkeitsmerkmale und der partnerschaftlichen Bindungsangst und vermeidung. In einer Längsschnittstudie über acht Monate zeigen sie, dass die Bindung durch die Big Five der Persönlichkeit prognostiziert werden kann. Neurotizismus erwies sich als signifikanter positiver Prädiktor des ängstlichen Bindungs- 
stils, während der vermeidende Bindungsstil vor allem durch Verträglichkeit (negativer Prädiktor) aber auch noch durch Neurotizismus (positiver Prädiktor) erklärt wird.

Hinsichtlich der kausalen Wirkung der Persönlichkeit auf Beziehungsmerkmale kommen Karney und Bradbury (1995) aufgrund ihrer Meta-Analyse zu dem Schluss, dass die Befunde zum Einfluss der Persönlichkeit auf Merkmale der Partnerschaft nicht konsistent sind. Allerdings zeigt sich in mehreren Studien, dass Neurotizismus, Impulsivität, Unverträglichkeit und mangelnde Gewissenhaftigkeit die Qualität und die Stabilität der Partnerschaft beeinträchtigen (u.a. Neyer 2003). Dabei erweist sich der Einfluss von Persönlichkeitsmerkmalen auf soziale Beziehungen als stärker, als umgekehrt der Einfluss von Beziehungserfahrungen auf Persönlichkeitsmerkmale (Asendorpf/Wilpers 1998).

\section{Fragestellung und Hypothesen}

Mit unserer Studie möchten wir prüfen, wie stabil die partnerbezogenen Bindungsdimensionen Angst und Vermeidung sind und ob sie über einen Zeitraum von acht Monaten weniger stabil sind als inhaltlich nahe Persönlichkeitsmerkmale. Wir versuchen zu beantworten, inwieweit die Bindung an einen Partner von wahrgenommenen Veränderungen, die im Beziehungsleben auftreten, beeinflusst wird. Darüber hinaus verfolgen wir das Ziel, den Zusammenhang zwischen Persönlichkeit und Bindung auf verschiedenen Abstraktionsebenen zu analysieren. Da von der allgemeinen Bindung angenommen wird, dass sie eher übergreifend in verschiedenen Beziehungen wirksam ist, kann vermutet werden, dass sie auch stärker mit stabilen übergreifenden Persönlichkeitsmerkmalen zusammenhängt als die partnerbezogene Bindung. Letztere sollte in stärkerem Ausmaß von beziehungsspezifischen Erfahrungen bestimmt werden. Aufgrund bisheriger Befunde nehmen wir an, dass die als weitgehend stabil konzipierten grundlegenden Persönlichkeitseigenschaften Neurotizismus und Verträglichkeit der Big Five besser die partnerbezogenen Bindungsdimensionen vorhersagen als umgekehrt die partnerbezogenen Bindungsdimensionen die Persönlichkeit.

Aus dem bisher Ausgeführten lassen sich die folgenden konkreten Hypothesen ableiten:

Hypothese 1: Die partnerbezogene Bindungsdimension Vermeidung erweist sich über einen Zeitraum von acht Monaten stabiler als die partnerbezogene Bindungsangst.

Hypothese 2: Die partnerbezogenen Bindungsdimensionen sind über acht Monate weniger stabil als inhaltlich ähnliche Persönlichkeitsmerkmale.

2a. Partnerbezogene Bindungsangst ist weniger stabil als Neurotizismus.

2b. Partnerbezogene Bindungsvermeidung ist weniger stabil als Verträglichkeit.

Hypothese 3: Die partnerbezogenen Bindungsdimensionen Angst und Vermeidung werden durch Veränderungen im Beziehungsleben beeinflusst. 
Hypothese 4: Die Bewertung der Beziehungsveränderungen beeinflusst die partnerbezogenen Bindungsdimensionen Angst und Vermeidung.

4a. Positiv bewertete Änderungen im Beziehungserleben gehen mit einer Abnahme von Angst und Vermeidung einher.

4b. Negativ bewertete Änderungen im Beziehungserleben gehen mit einer $\mathrm{Zu}-$ nahme von Angst und Vermeidung einher.

Hypothese 5: Die generellen Persönlichkeitsmerkmale sind bessere Prädiktoren für die allgemeinen Bindungsdimensionen als für die partnerbezogenen Bindungsdimensionen.

5a. Neurotizismus sagt besser die allgemeine Bindungsangst als die partnerbezogene Bindungsangst vorher.

5b. Verträglichkeit sagt besser die allgemeine Bindungsvermeidung als die partnerbezogene Bindungsvermeidung vorher.

Hypothese 6: Die Einflüsse der Persönlichkeitsmerkmale auf die partnerbezogenen Bindungsdimensionen sind stärker, als die Einflüsse der Bindungsdimensionen auf Persönlichkeitsmerkmale.

6a. Der Einfluss von Neurotizismus auf partnerbezogene Bindungsangst ist stärker, als der Einfluss von partnerbezogener Bindungsangst auf den Neurotizismus.

6b. Der Einfluss von Verträglichkeit auf die partnerbezogene Bindungsvermeidung ist stärker, als der Einfluss der partnerbezogenen Bindungsvermeidung auf Verträglichkeit.

\section{$4 \quad$ Methode}

Wir befragten Psychologie-StudentInnen der Ruhr-Universität Bochum im Längsschnitt über acht Monate mit insgesamt drei Messzeitpunkten.

\subsection{Beschreibung der Messinstrumente}

Die folgenden demographischen Variablen wurden zu allen drei Messzeitpunkten erfasst: Geschlecht, Alter, Familienstand, Beziehungsstatus (Single, feste Beziehung oder verheiratet) und Wohnsituation. Bei bestehender Partnerschaft wurde zudem die Beziehungsdauer erfragt. Zum ersten Messzeitpunkt wurde erfasst, ob sich im letzten halben Jahr die Lebenssituation der Probanden deutlich verändert habe. Falls dies der Fall war, sollten sie mit eigenen Worten beschreiben, inwiefern sich ihre Lebenssituation verändert hat. Zum zweiten Messzeitpunkt wurden die Probanden gefragt, ob die zum ersten Messzeitpunkt (drei Monate zuvor) geführte Beziehung noch besteht und ob sich ihre Lebenssituation seit der letzten Befragung stark verändert hat. Die Probanden, die diese Frage bejahten, sollten wiederum angeben, inwiefern sich ihre Lebenssituation verändert hat. Hierfür wurden 
drei Kategorien vorgegeben: Ortswechsel, Änderungen im Beruf/Studium und Veränderungen im Beziehungsleben (z.B. Trennung/neue(r) Partner(in)), Mehrfachnennungen waren möglich. Die vorgegebenen Kategorien basierten auf den offenen Antworten zur Veränderung von Lebenssituation des ersten Messzeitpunkts. Da sich diese Messung der Veränderung im Beziehungsleben zum zweiten Messzeitpunkt als zu undifferenziert erwies, wurde zum dritten Messzeitpunkt ein Rating zur Erfassung der Veränderung im Beziehungsleben vorgegeben (Frage: „Inwieweit hat sich seit der letzten Befragung Ihr Beziehungserleben verändert?“). Dieses Item wurde auf einer Rating-Skala von gar nicht (1) bis sehr (7) beurteilt. Zudem wurde anhand einer sieben-stufigen bipolaren Skala summarisch erfasst, wie diese Beziehungsveränderungen bewertet wurden. Die Frage lautete: „Insgesamt finde ich diese Beziehungsveränderungen sehr negativ (-3) bzw. sehr positiv $(+3)^{*}$.

Die folgenden Messinstrumente wurden zur Erfassung von Bindung und Persönlichkeit eingesetzt:

Es wird argumentiert, dass eine kontinuierliche (statt kategoriale) Erfassung der Dynamik des Bindungssystems besser gerecht wird (Scharfe/Bartholomew 1994; Fraley/Waller 1998). Daher wurde die partnerbezogene Bindung mit Hilfe der Skalen zur Erfassung von Bindungsrepräsentationen in Paarbeziehungen von Grau (1999) erhoben. Dieser Fragebogen misst die beiden Dimensionen Angst (10 Items) und Vermeidung (10 Items). Wir erfassten die generelle partnerbezogene Bindung. Mit dieser beziehungsspezifischen aber nicht zielpersonenspezifischen Messung konnten auch Singles berücksichtigt werden. Ein Beispiel-Item für Angst lautet: "Ich habe Angst, dass ein Partner/eine Partnerin die Beziehung zu mir abbricht.“ Ein Beispiel-Item für Vermeidung lautet: „Ich bin gewöhnlich lieber allein, als mit einem/einer Partnerin zusammen." Die Befragten beurteilten die zwanzig Aussagen jeweils auf einer sieben-stufigen Skala mit den Endpunkten „stimmt nicht“" und „stimmt völlig“.

Die Erfassung der allgemeinen Bindung erfolgte in einer Version von Grau (1999), in der die 20 Items des Fragebogens ohne Bezug auf einen Partner formuliert sind und ebenfalls mit einer 7-stufigen Skala erfasst werden (z.B. „Ich bin gewöhnlich lieber allein, als mit anderen zusammen."). Zur besseren Abgrenzung der Konstrukte und um systematische Reihenfolge-Effekte zu verhindern, wurde darauf geachtet, dass die Abfrage der allgemeinen und partnerbezogenen Bindung nicht direkt aufeinander folgte und in der Reihenfolge variierte. ${ }^{1}$

Obwohl in der Skala zur Erfassung der allgemeinen Bindung eine recht heterogene Gruppe von Zielpersonen (Menschen allgemein, Freunde oder Bekannte) angesprochen wird, weisen die beiden Sub-Skalen allgemeine Bindungsangst und allgemeine Bindungsvermeidung bei Grau (1994) gute interne Konsistenzen auf $(=.80$ bis $=.86)$, was indirekt ein Hinweis auf eine gewisse Invarianz des Bindungserlebens in verschiedenen Beziehungen ist und auf eine generelle Bindungs-

1 Version A: Demografische Variablen, Lebens- und Beziehungsveränderungen, partnerbezogene Bindung, Persönlichkeit, allgemeine Bindung. Version B: Allgemeine Bindung, Persönlichkeit, partnerbezogene Bindung, demografische Variablen, Lebens- und Beziehungsveränderungen. 
orientierung hindeutet. Die auf diese Weise dimensional erfasste allgemeine Bindung hängt deutlich mit der partnerbezogenen Bindung zusammen $(\mathrm{r}=.33$ bis $r=.70$ ). Allerdings korreliert die allgemeine Bindung höher mit Personmerkmalen, die partnerbezogene Bindung hingegen höher mit Merkmalen (z.B. Glück) der Partnerschaft (Grau, 1994).

Neurotizismus und Verträglichkeit wurden mit den entsprechenden Skalen des NEO-FFI von Borkenau und Ostendorf (1993) erfasst. Neurotizismus: Personen mit hohen Werten neigen dazu nervös, ängstlich und unsicher zu sein; haben unrealistische Ideen; reagieren unangemessen auf Stresssituationen und werden von negativen Gefühlszuständen manchmal überwältigt. Verträglichkeit: Personen mit hohen Werten sind altruistisch, mitfühlend und harmoniebedürftig; neigen zu Vertrauen, Kooperativität und Nachgiebigkeit. Beide Dimensionen wurden jeweils mit 12 Items erfasst, die jeweils anhand eines 5-stufigen Ratings ( $1=$ starke Ablehnung, 2 = Ablehnung, $3=$ Neutral, $4=$ Zustimmung, $5=$ starke Zustimmung) beurteilt werden sollten.

\subsection{Stichprobe}

Die Stichprobe wurde in Pflichtveranstaltungen der Sozialpsychologie während der ersten beiden Semester rekrutiert. Die drei Erhebungszeitpunkte fanden innerhalb von acht Monaten statt, wobei der erste Messzeitpunkt zu Beginn des ersten Semesters, der zweite Messzeitpunkt drei Monate später am Ende des ersten Semesters und der dritte Messzeitpunkt am Ende des zweiten Semesters weitere fünf Monate später lag.

Am ersten Messzeitpunkt nahmen 121 Studierende teil (27 Männer, 94 Frauen), die im Mittel 26 Jahre alt waren (Minimum = 19, Maximum = 51). 67 lebten in einer festen Beziehung, 33 waren verheiratet und 21 Single. Am zweiten Messzeitpunkt nahmen noch 100 Personen teil (82.6\%; 82 Frauen und 18 Männer). 57 lebten in einer festen Beziehung, 16 waren verheiratet und 27 Singles. Am dritten Messzeitpunkt nahmen noch 57 Personen teil $(47.1 \%$ von den 121 Studierenden des ersten Messzeitpunktes; 45 Frauen und 12 Männer). Von den 57 Personen lebten 34 in einer festen Beziehung, 8 waren verheiratet und 14 waren Singles (eine Angabe fehlte).

Die Stichprobe umfasste also 57 Personen, die zu allen drei Messzeitpunkten befragt werden konnten. Diejenigen, die zum zweiten bzw. dritten Messzeitpunkt (MZP) nicht mehr befragt werden konnten, waren entweder nur am Befragungstag nicht da, hatten nicht mehr am Seminar teilgenommen oder waren möglicherweise sogar ganz aus dem Studium ausgestiegen. In der Tendenz waren diejenigen, die bereits nach der ersten oder zweiten Messung aus der Stichprobe heraus gefallen waren, älter und signifikant häufiger männlich $\left(\mathrm{Chi}^{2}(\mathrm{df}=2)=6.89, \mathrm{p}<.05\right)$ als diejenigen, die bis zum dritten Messzeitpunkt teilgenommen hatten. Keine Unterschiede zwischen den Gruppen zeigten sich hingegen beim Familienstand, dem Vorhandensein von Kindern und dem Beziehungsstatus (Single oder festen Partner). Besonders wichtig für die Bewertung der Ergebnisse ist, dass die Gruppen sich nicht in der Bindungsangst oder -vermeidung unterschieden $(F(4,234)=$ 
.310 , n.s.) und auch nicht in den erlebten Lebensveränderungen vor dem ersten Messzeitpunkt $\left(\mathrm{Ch}^{2}(\mathrm{df}=2)=2.41 \mathrm{n} . \mathrm{s}\right)$. Zudem unterschieden sich die drei Gruppen nicht in ihrer Erfahrung mit kürzeren (höchstens sechs Monate) und längeren Beziehungen (mindestens sechs Monate); F $(4,148)=.894$, n.s.

Mit der Länge der Teilnahme stieg lediglich die Angabe von Veränderungen im Berufsleben marginal $\left(\mathrm{Chi}^{2}(\mathrm{df}=2)=5.67, \mathrm{p}<.10\right)$.

Die hohe drop-out-Quote sowie der Überhang männlicher Abbrecher in unserer Stichprobe ist leicht erklärbar, betrachtet man die hohe Quote von Studienabbrechern und Fachwechslern von bis zu 68\% bei Erstimmatrikulierten in den Geistesund Sozialwissenschaften. Dabei brechen mehr Männer als Frauen ihr Studium ab (Heublein/Schmelzer/Sommer 2005). Der Ausstieg aus der Befragung scheint also eher etwas mit dem Studium als mit dem Beziehungsleben zu tun zu haben. Insgesamt lässt sich festhalten, dass die drei Stichproben in den für die Untersuchung wesentlichen beziehungsrelevanten Merkmalen keine substantiellen Unterschiede aufwiesen.

\section{Ergebnisse}

\subsection{Interne Konsistenzen und Interkorrelationen}

Die internen Konsistenzen (Cronbachs ) der partnerbezogenen Bindung sind zu allen drei Messzeitpunkten sowohl für die Dimension Angst ( $=.87$ bis $=.92$ ) als auch für die Dimension Vermeidung $(=.82-.88)$ sehr überzeugend. Dies gilt ebenfalls für die nur zum dritten Messzeitpunkt erfasste allgemeine Bindungsangst ( $=.87$ ) und allgemeine Bindungsvermeidung $(=.83)$. Die internen Konsistenzen sind für Neurotizismus ( $=.82$ bis $=.88$.) gut und für Verträglichkeit ${ }^{2}(=.65$ bis $=.71)$ zufrieden stellend.

Wie Tabelle 1 mit den Interkorrelationen der Skalen zu den jeweiligen Messzeitpunkten zeigt, hängen die Dimensionen partnerbezogene Angst und Vermeidung nur zum dritten Messzeitpunkt zusammen $(r=.30, \mathrm{p}<.05)$. Die partnerbezogene Vermeidung hängt zu allen drei Messzeitpunkten negativ mit Verträglichkeit zusammen ( $\mathrm{t} 1 \mathrm{r}=-.34, \mathrm{p}<.01 ; \mathrm{t} 2 \mathrm{r}=-.40, \mathrm{p}<.01 ; \mathrm{t} 3 \mathrm{r}=-.39, \mathrm{p}<.01)$, ebenso wie die allgemeine Vermeidung, die zum dritten Messzeitpunkt erfasst wurde $(\mathrm{r}=$ $-.42, \mathrm{p}<.01)$. Partnerbezogene Angst hängt zum ersten und zweiten Messzeitpunkt mit Neurotizismus zusammen $(\mathrm{r}=.34, \mathrm{p}<.01$ und $\mathrm{r}=.25, \mathrm{p}<.05)$; zum dritten Messzeitpunkt ist die Korrelation mit $\mathrm{r}=.24$ nicht signifikant. Die allgemeine Angst hingegen korreliert zum dritten Messzeitpunkt hoch mit Neurotizismus ( $\mathrm{r}=$ $.59, \mathrm{p}<.01)$. Der Unterschied zwischen den beiden Korrelationen ist bei einsei-

2 Item 4 der Skala Verträglichkeit wurde aufgrund der geringen Trennschärfe beim dritten Messzeitpunkt $\left(r_{i t}=.07\right)$ ausgeschlossen, sodass die Skala Verträglichkeit 11 Items umfasst. 
tiger Testung signifikant $(\mathrm{z}=3.25, \mathrm{p}<.01){ }^{3}$ Sowohl partnerbezogene und allgemeine Angst $(\mathrm{r}=.69, \mathrm{p}<.001)$ als auch partnerbezogene und allgemeine Vermeidung $\quad(\mathrm{r}=.87, \mathrm{p}<.001)$ korrelieren miteinander.

Tabelle 1: $\quad$ Interkorrelationen der Skalen innerhalb der Messzeitpunkte

\begin{tabular}{|c|c|c|c|}
\hline \multirow[t]{2}{*}{ Variablen } & \multicolumn{3}{|c|}{ Messzeitpunkte } \\
\hline & $\mathrm{t} 1$ & t 2 & t3 \\
\hline P-Angst/P-Vermeidung & .05 & .13 & $.30^{*}$ \\
\hline P-Angst/Neurotizismus & $.34^{\star \star}$ & $.25^{*}$ & .24 \\
\hline P-Angst/Verträglichkeit & $-.27^{\star \star}$ & -.11 & -.12 \\
\hline P-Vermeidung/Neurotizismus & .02 & -.01 & .04 \\
\hline P-Vermeidung/Verträglichkeit & $-.34^{\star \star}$ & $-.40^{\star \star}$ & $-.39^{\star \star}$ \\
\hline Verträglichkeit/Neurotizismus & -.10 & -.07 & -.01 \\
\hline A-Angst/A-Vermeidung & & & .19 \\
\hline P-Angst/A-Angst & & & $.69^{\star \star}$ \\
\hline P-Vermeidung/A-Vermeidung & & & $.87^{\star \star}$ \\
\hline P-Angst/A-Vermeidung & & & $.28^{*}$ \\
\hline P-Vermeidung/A-Angst & & & .20 \\
\hline A-Angst/Neurotizismus & & & $.59^{\star \star}$ \\
\hline A-Angst/Verträglichkeit & & & -.17 \\
\hline A-Vermeidung/Neurotizismus & & & -.01 \\
\hline A-Vermeidung/Verträglichkeit & & & $-.42^{\star \star}$ \\
\hline
\end{tabular}

\subsection{Stabilität der partnerbezogenen Bindungsangst und Bindungsvermeidung im Vergleich}

Die Retest-Reliabilität von Bindungsangst über acht Monate liegt bei $r_{t t}=.60$, die für Bindungsvermeidung bei $r_{t t}=.68$ (siehe Tabelle 2). Die beiden Korrelationen unterscheiden sich nicht signifikant $(\mathrm{z}=.72) .{ }^{4}$ Damit lässt sich Hypothese 1 , die annimmt, dass sich partnerbezogene Bindungsvermeidung als stabiler erweist als partnerbezogene Bindungsangst, nicht bestätigen.

\subsection{Stabilität der Bindungs- und Persönlichkeitsdimensionen im Vergleich}

Wir prüften Hypothese 2 folgend, die Stabilität der Bindungsdimensionen im Vergleich zur Stabilität von Neurotizismus und Verträglichkeit.

3 Berechnung auf Basis der empfohlenen Formel von Bortz (1993: 205) zum Vergleich von überlappenden Korrelationskoeffizienten. Der kritische $z$-Wert bei einseitiger Testung auf einem $1 \%$-Niveau liegt bei $\mathrm{z}=2.33$.

4 Berechnung auf der Basis der empfohlenen Formel von Raghunathan, Rosenthal und Rubin (1996) zum Vergleich von nicht überlappenden Korrelationskoeffizienten. Der kritische $\mathrm{z}$-Wert bei einseitiger Testung auf einem 5\%-Niveau liegt bei $\mathrm{z}=1.65$ und auf dem $1 \%$-Niveau bei $\mathrm{z}=2.33$. 
Die Retest-Reliabilitäten der Bindungs- und der Persönlichkeitsdimensionen sind in Tabelle 2 dargestellt. Mit $r_{t t}=.68$ und $r_{t t}=.67$ sind die Retest-Reliabilitäten von Vermeidung und Verträglichkeit über acht Monate nahezu identisch. Auch der Retest Angst $\left(\mathrm{r}_{\mathrm{tt}}=.60\right)$ ist vom Retest Neurotizismus $\left(\mathrm{r}_{\mathrm{tt}}=.73\right)$ über diesen Zeitraum nicht signifikant verschieden $(\mathrm{z}=1.09)$. Damit lässt sich Hypothese 2, die eine geringere Stabilität der partnerbezogenen Bindungsdimensionen im Vergleich zu den Persönlichkeitsdimensionen postuliert, nicht bestätigen.

Tabelle 2: $\quad$ Retest-Reliabilitäten der partnerbezogenen Bindungsdimensionen Angst und Vermeidung und der Persönlichkeitseigenschaften Neurotizismus und Verträglichkeit (Pearson-Korrelationen)

\begin{tabular}{||l|c|c|c||}
\hline \hline Variablen & $\mathrm{r}(\mathrm{MZP} 1 / 2)$ & $\mathrm{r}(\mathrm{MZP} 2 / 3)$ & $\mathrm{R}(\mathrm{MZP} 1 / 3)$ \\
\hline Angst & $.77(\mathrm{~N}=99)$ & $.75(\mathrm{~N}=57)$ & $.60(\mathrm{~N}=57)$ \\
\hline Vermeidung & $.83(\mathrm{~N}=99)$ & $.78(\mathrm{~N}=57)$ & $.68(\mathrm{~N}=57)$ \\
\hline Neurotizismus & $.85(\mathrm{~N}=100)$ & $.79(\mathrm{~N}=57)$ & $.73(\mathrm{~N}=57)$ \\
\hline Verträglichkeit & $.74(\mathrm{~N}=100)$ & $.84(\mathrm{~N}=57)$ & $.67(\mathrm{~N}=57)$ \\
\hline
\end{tabular}

Anmerkung: MZP 1/2: zeitlicher Abstand drei Monate; MZP 2/3: zeitlicher Abstand fünf Monate; MZP 1/3: zeitlicher Abstand acht Monate.

\subsection{Partnerbezogene Bindungsdimensionen und Beziehungsveränderungen}

Um zu prüfen, inwieweit Beziehungsveränderungen einen Einfluss auf die partnerbezogenen Bindungsdimensionen haben (Hypothese 3), berechneten wir Partialkorrelationen zwischen dem Ausmaß, in dem Beziehungsveränderungen angegeben wurden, und den beiden Bindungsdimensionen. Entsprechend wurden die zum dritten Messzeitpunkt angegebenen Beziehungsveränderungen $(1=$ gar nicht bis 7 $=$ sehr) mit der Bindungsangst des gleichen Messzeitpunkts korreliert, wobei die Bindungsangst des zweiten Messzeitpunkts bei der Berechnung der Korrelation herauspartialisiert wurde. Entsprechend wurde bei der Bindungsvermeidung verfahren. Sowohl die Bindungsangst $(r=.15$, n.s., $N=53$ ) als auch die Bindungsvermeidung $(r=.03$, n.s., $\mathrm{N}=53$ ) stehen in keinem Zusammenhang mit Beziehungsveränderungen.

Es ist jedoch nahe liegend, zu vermuten, dass die Bewertung der Beziehungsveränderungen einen Einfluss auf Angst und Vermeidung haben (Hypothese 4a und 4b). Daher wurden Partialkorrelationen zwischen Beziehungsveränderungen und Angst bzw. Vermeidung berechnet, wobei nur diejenigen einbezogen werden konnten, die Beziehungsveränderungen angaben $(\mathrm{N}=42)$. Die Bewertung der Beziehungsveränderungen wurde summarisch bipolar erfasst. 12 Probanden bewerteten die Beziehungsveränderungen als neutral (0), 29 Probanden bewerteten die Beziehungsveränderungen als positiv $(+1$ bis +3$)$ und 7 Probanden als negativ $(-1$ bis -2). Um den Zusammenhang zwischen positiven Beziehungsveränderungen und Bindungsangst bzw. Bindungsvermeidung einerseits und negativen Beziehungsveränderungen und Angst und Vermeidung andererseits festzustellen, wurden zwei Gruppen gebildet. In Anlehnung an die Vorgehensweise von Sprecher (1986) wur- 
den für die Analyse des Zusammenhangs zwischen den Bindungsdimensionen und positiven Beziehungsveränderungen diejenigen einbezogen, die die Beziehungsveränderungen neutral $(0)$ bis sehr positiv $(+3)$ beurteilten. Zur Berechnung der negativen Bewertung der Beziehungsveränderungen wurden diejenigen einbezogen, die die Beziehungsveränderungen von neutral (0) bis sehr negativ (-2) bewerteten.

Die Berechnung der Partialkorrelation Beziehungsveränderungen/Bindungsangst zum dritten Messzeitpunkt für Personen mit neutralen bzw. positiven Beziehungsveränderungen mit Bindungsangst des zweiten Messzeitpunkts als Kontrollvariable zeigt mit $\mathrm{r}=-.36(\mathrm{p}<.05, \mathrm{~N}=41)$ bei einseitiger Testung einen signifikanten Zusammenhang. Dieses Ergebnis ist auch dann gegeben, wenn nur diejenigen in die Analyse einbezogen werden, die die Beziehungsveränderungen positiv bewertet haben, $\mathrm{r}=-.44(\mathrm{p}=.01, \mathrm{~N}=29)$. Je positiver die Beziehungsveränderungen bewertet werden, desto geringer ist die Bindungsangst. Für die Personen mit positiv bzw. neutral bewerteten Beziehungsveränderungen liegt zwischen der Beziehungsveränderungen und Bindungsvermeidung des dritten Messzeitpunkts - bei Kontrolle der Bindungsvermeidung des zweiten Messzeitpunkts kein Zusammenhang vor $\quad(r=-.25$, n.s., $\mathrm{N}=41)$. Dieser zeigt sich auch dann nicht, wenn nur diejenigen in die Analyse einbezogen werden, die die Beziehungsveränderungen positv bewertet haben $(r=-.18$, n.s., $\mathrm{N}=29)$.

Für diejenigen, die die Beziehungsveränderungen negativ bzw. neutral bewertet haben, zeigt sich bei einseitiger Testung ein negativer Zusammenhang zwischen der Bewertung der Beziehungsveränderungen und Angst bei Kontrolle der Angst des zweiten Messzeitpunkts ( $\mathrm{r}=-.67, \mathrm{p}<.01, \mathrm{~N}=19)$, der auch dann vorhanden ist, wenn nur diejenigen in die Analyse einbezogen werden, die negative Beziehungsveränderungen angegeben haben $(\mathrm{r}=-.80, \mathrm{p}<.01, \mathrm{~N}=7)$. Je negativer die Beziehungsveränderungen bewertet werden, desto stärker ist die Bindungsangst ausgeprägt. Für Personen, die die Beziehungsveränderungen negativ bzw. neutral bewerteten, ist die Korrelation zwischen Bewertung der Beziehungsveränderungen und Bindungsvermeidung bei Kontrolle der Bindungsvermeidung des zweiten Messzeitpunkts nahezu null $(\mathrm{r}=-.09$, n.s., $\mathrm{N}=19)$. Die Korrelation wird auch dann nicht signifikant, wenn nur diejenigen in die Analyse einbezogen werden, die negative Beziehungsveränderungen angegeben haben $(r=-.30$, n.s., $N=7)$.

Für Hypothese 3 kann festgehalten werden, dass Beziehungsveränderungen allein nicht mit Veränderungen der partnerbezogenen Bindungsdimensionen einhergehen. Deutlich wird vielmehr, dass es auf die Bewertung dieser Veränderungen ankommt (Hypothese 4): Bindungsangst nimmt wie erwartet (Hypothese 4 b) mit positiv bewerteten Beziehungsveränderungen ab und mit negativ bewerteten Veränderungen in der Beziehung zu. Die Bindungsvermeidung hingegen steht in keinem Zusammenhang mit negativ und positiv bewerteten Veränderungen in der Beziehung. 


\subsection{Bindungsdimensionen und Persönlichkeitsdimensionen}

Es wurde geprüft, inwieweit Neurotizismus und Verträglichkeit eher die allgemeinen als die partnerbezogenen Bindungsdimensionen vorhersagen können (Hypothese 5). Dazu wurden vier schrittweise multiple Regressionsanalysen mit den abhängigen Variablen allgemeine Bindungsangst, partnerbezogene Bindungsangst, allgemeine Bindungsvermeidung und partnerbezogene Bindungsvermeidung (alle dritter Messzeitpunkt) gerechnet. Als Prädiktoren wurden jeweils Neurotizismus und Verträglichkeit des ersten Messzeitpunkts eingesetzt.

Tabelle 3: $\quad$ Vorhersage von partnerbezogener (P-Angst) und allgemeiner Bindungsangst (A-Angst) bzw. partnerbezogener (P-Vermeidung) und allgemeiner Bindungsvermeidung (A-Vermeidung) des dritten Messzeitpunkts durch Neurotizismus und Verträglichkeit des ersten Messzeitpunkts

\begin{tabular}{||l|c|c|c|c|c|c|c|c||}
\hline Variable & $\mathrm{R}$ & $\mathrm{Beta}$ & $\mathrm{T}$ & $\mathrm{p}$ & $\mathrm{R}$ & $\mathrm{R}^{2}$ & $\mathrm{Df}$ & $\mathrm{F}$ \\
\hline P-Angst durch & & & & & .34 & .11 & 1,55 & $7.09^{\star *}$ \\
\hline Neurotizismus & $.34^{\star *}$ & $.34^{\star \star}$ & 2.66 & .010 & & & & \\
\hline $\begin{array}{l}\text { Ausgeschlossene } \\
\text { Variablen }\end{array}$ & & Beta In & & & & & & \\
\hline Verträglichkeit & -.16 & -.14 & -1.09 & .280 & & & & \\
\hline A-Angst durch & & & & & .73 & .53 & 1,55 & $62.3^{\star *}$ \\
\hline Neurotizismus & $.73^{\star *}$ & $.73^{\star *}$ & 7.90 & .000 & & & & \\
\hline $\begin{array}{l}\text { Ausgeschlossene } \\
\text { Variablen }\end{array}$ & & Beta In & & & & & & \\
\hline Verträglichkeit & -.07 & -.03 & -.31 & .756 & & & & \\
\hline $\begin{array}{l}\text { P-Vermeidung } \\
\text { durch }\end{array}$ & & & & & .47 & .22 & 1,55 & $15.19^{\star *}$ \\
\hline Verträglichkeit & $-.47^{\star *}$ & $-.47^{\star *}$ & -3.99 & .001 & & & & \\
\hline $\begin{array}{l}\text { Ausgeschlossene } \\
\text { Variablen }\end{array}$ & & Beta In & & & & & & \\
\hline Neurotizismus & .05 & .02 & .19 & .852 & & & & \\
\hline $\begin{array}{l}\text { A-Vermeidung } \\
\text { durch }\end{array}$ & & & & & 46 & .22 & 1,55 & $15.12^{* *}$ \\
\hline Verträglichkeit & $-.46^{\star *}$ & $-.46^{\star *}$ & -3.88 & .001 & & & & \\
\hline $\begin{array}{l}\text { Ausgeschlossene } \\
\text { Variablen }\end{array}$ & & Beta In & & & & & & \\
\hline Neurotizismus & .04 & -.00 & -.02 & .983 & & & & \\
\hline
\end{tabular}

${ }^{\star} \mathrm{p}<.05 ;{ }^{* *} \mathrm{p}<.001$.

Aus Tabelle 3 ist ersichtlich, dass sowohl die partnerbezogene als auch die allgemeine Bindungsangst durch den Prädiktor Neurotizismus vorhergesagt werden können. Die Varianzaufklärung von allgemeiner Bindungsangst durch Neurotizismus fällt mit 53\% im Vergleich zu partnerbezogener Bindungsangst mit 11\% besonders hoch aus. Der Vergleich der Korrelation von Neurotizismus und partnerbezogener Bindungsangst $(\mathrm{r}=.34)$ einerseits mit der Korrelation von Neurotizismus und allgemeiner Bindungsangst $(r=.73)$ andererseits zeigt, dass die Zusammenhänge signifikant verschieden sind $(\mathrm{z}=3.97, \mathrm{p}<.01$ bei einseitiger Testung; 
siehe Fußnote 2). Neurotizismus sagt die allgemeine Bindungsangst nach acht Monaten besser vorher als die partnerbezogene Bindungsangst.

Aus Tabelle 3 ist ersichtlich, dass die Verträglichkeit die partnerbezogene Bindungsvermeidung mit einer Varianzaufklärung von $22 \%$ genauso gut erklärt wie die allgemeine Bindungsvermeidung.

Es bleibt in Bezug auf Hypothese 5 festzuhalten, dass sowohl die partnerbezogene Bindungsangst als auch die allgemeine Bindungsangst durch Neurotizismus vorhergesagt werden können. Hypothesenkonform erklärt Neurotizismus die allgemeine Bindungsangst besser als die partnerbezogene Bindungsangst (Hypothese 5 a). Die Varianzaufklärung von allgemeiner und partnerbezogener Bindungsvermeidung durch Verträglichkeit ist dagegen identisch (Hypothese $5 \mathrm{~b}$ ).

Die Wirkungsrichtung von Bindung und Persönlichkeit (siehe Hypothese 6) prüften wir mit Hilfe einer cross-lagged panel-Analyse (Kenny, 1975). Von zentraler Bedeutung für die Beantwortung nach kausaler Priorität (vgl. Rudinger/Bierhoff 1980) sind die verzögerten Kreuzkorrelationen. Die beiden Autokorrelationen $\mathrm{r}$ (Neurotizismus 1. MZP, Neurotizismus 3. MZP) und $\mathrm{r}$ (Bindungsangst 1. MZP, Bindungsangst 3. MZP) und die synchronen Korrelationen $r$ (Neurotizismus 1. MZP, Bindungsangst 1. MZP) und r (Neurotizismus 3. MZP, Bindungsangst 3. MZP) bestimmen den interpretativen Kontext.

Abbildung 1: Korrelationen zwischen partnerbezogener Bindung und Neurotizismus innerhalb und zwischen den Messzeitpunkten $(\mathrm{N}=57)$

$.73^{\star \star}$

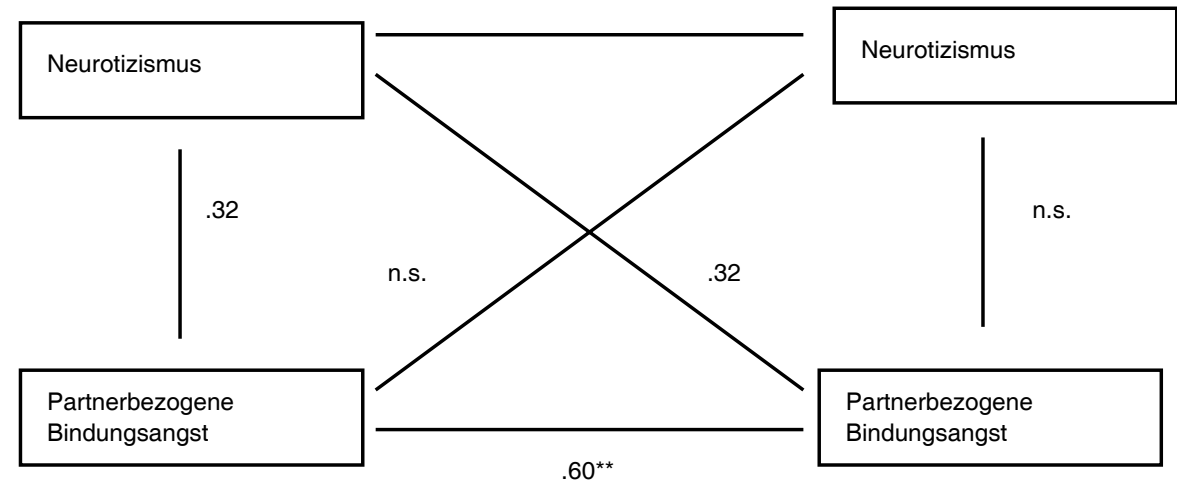

${ }^{* *} p<.01,{ }^{*} p<.05$

Wie in Abbildung $1 \mathrm{zu}$ sehen ist, korreliert Neurotizismus (1. MZP) signifikant mit partnerbezogener Bindungsangst (3. MZP). Dagegen ist die Korrelation zwischen 
partnerbezogener Bindungsangst (1. MZP) und Neurotizismus (3. MZP) nicht signifikant.

Dieses Ergebnis weist darauf hin, dass Neurotizismus eher die partnerbezogene Bindungsangst beeinflusst als umgekehrt. Die Korrelation zwischen Verträglichkeit (1. MZP) und partnerbezogener Bindungsvermeidung (3. MZP) fällt mit $r=-$ $.47(\mathrm{p}<.01)$ relativ hoch aus. Der Zusammenhang zwischen Bindungsvermeidung (1. MZP) und Verträglichkeit (3. MZP) liegt bei $r=-.35$ und ist mit $p<.01$ ebenso signifikant. Hypothese 6 a wird bestätigt, da partnerbezogene Bindungsangst durch Neurotizismus vorhergesagt wird, nicht aber Neurotizismus durch Bindungsangst. Für Bindungsvermeidung und Verträglichkeit kann die Richtung des Einflusses nicht festgelegt werden (Hypothese 6b).

\section{Diskussion}

Wie in den Hypothesen angenommen, besteht ein Zusammenhang zwischen Persönlichkeitsdimensionen und Bindungsdimensionen: Bindungsangst lässt sich durch Neurotizismus und Bindungsvermeidung durch Verträglichkeit vorhersagen. Diese Ergebnisse sind mit den Befunden von Shaver und Brennan (1992) im Einklang, die fanden, dass Neurotizismus die ängstliche Bindung vorhersagt und sich vor allem Verträglichkeit als negativer Prädiktor des vermeidenden Bindungsstils erweist.

Darüber hinaus zeigt unsere Studie, dass wie erwartet über den Zeitraum von acht Monaten die allgemeine Bindungsangst durch Neurotizismus besser vorhergesagt wird als die partnerbezogene Bindungsangst. Neurotizismus kommt somit mehr in der allgemeinen Bindungsangst, die viele Beziehungen betrifft, zum Ausdruck als in der beziehungsspezifischen Bindungsangst. Die allgemeine und partnerbezogene Bindungsvermeidung lassen sich hingegen gleichermaßen gut durch die Verträglichkeit vorhersagen. Im Vergleich zur partnerbezogenen Bindungsangst weist die partnerbezogene Bindungsvermeidung somit eine geringere Spezifität auf.

Unterstützt wird dieses Ergebnis durch die signifikant unterschiedliche Korrelation $(\mathrm{z}=2.76$, siehe Fußnote 3$)$ der partnerbezogenen Bindungsvermeidung mit allgemeiner Bindungsvermeidung $(\mathrm{r}=.87)$ im Vergleich $\mathrm{zu}$ partnerbezogener Angst mit allgemeiner Angst $(r=.69)$. Dieses Ergebnis ist in Einklang mit Grau (1999), die für die auf den aktuellen Partner bezogene Bindungsvermeidung (Korrelation mit allgemeiner Bindungsvermeidung $r=.71$ ) eine höhere Generalität als für die auf den aktuellen Partner bezogene Bindungsangst (Korrelation mit allgemeiner Bindungsangst $r=.39$ ) findet.

Auch unsere cross-lagged-panel-Analyse zum Einfluss der Persönlichkeitsdimensionen auf die Bindungsdimensionen belegt die Notwendigkeit einer differenzierten Betrachtung von Bindungsangst und -vermeidung. Für die partnerschaftliche Bindungsangst zeigt sich, dass sie stärker vom Neurotizismus beeinflusst wird, als dass von der Bindungsangst eine Wirkung auf den Neurotizismus ausgeht. Für die partnerbezogene Bindungsangst spielt somit offenbar die generelle Neigung 
einer Person, auf Stress nervös und ängstlich zu reagieren und sich von Gefühlen überwältigen zu lassen, wie sie durch den Neurotizismus repräsentiert wird, eine Rolle. Der gefundene Einfluss von Neurotizismus auf die partnerbezogene Bindungsangst steht im Einklang mit Asendorpf und Wilpers (1998), die einen stärkeren Einfluss von Persönlichkeitsmerkmalen auf soziale Beziehungen fanden als umgekehrt. Einschränkend ist zu beachten, dass die kausale Dominanz von Neurotizismus auf Bindungsangst nicht bedeuten muss, dass kein wechselseitiger Einfluss zwischen Neurotizismus und Bindungsangst besteht, denn die Differenz zwischen den verzögerten Kreuzkorrelationen ist zwar signifikant, aber die Interpretierbarkeit dieses Ergebnisses wird durch die hohen Autokorrelationen und die fehlende Identität der beiden synchronen Korrelationen eingeschränkt (Rudinger/Bierhoff 1980). Festzuhalten bleibt jedoch, dass Neurotizismus einen stärkeren Einfluss auf die Bindungsangst ausübt als umgekehrt.

Im Gegensatz dazu zeigt sich keine dominante Wirkungsrichtung für den $\mathrm{Zu}$ sammenhang zwischen Verträglichkeit und partnerbezogener Bindungsvermeidung. Jedoch ist der Zusammenhang zwischen den beiden Konstrukten über den Zeitraum von acht Monaten relativ hoch (Verträglichkeit 1. MZP/Bindungsvermeidung 3. MZP $\mathrm{r}=-.47$; Bindungsvermeidung 1 . MZP/Verträglichkeit 3. MZP r = -.35). Einerseits verhalten sich Personen mit zunehmender Unverträglichkeit distanziert in der Partnerschaft, was darauf hinweist, dass die Distanz zum Partner durch dieses Persönlichkeitsmerkmal beeinflusst wird. Andererseits führt die distanzierte Haltung dem Partner gegenüber generell zu weniger Vertrauen und Empathie, wie es in der Unverträglichkeit zum Ausdruck kommt. Hier deutet sich ein Teufelskreis an: Durch das eigene distanzierte Verhalten (und möglicherweise auch das dadurch erzeugte Verhalten des Partners) scheinen die Eigenschaften, die die Unverträglichkeit beinhaltet, noch verstärkt zu werden, wodurch wiederum die Bindungsvermeidung verstärkt wird.

Die Retest-Reliabilitäten über einen Zeitraum von acht Monaten bestätigen nicht die von uns angenommene höhere Stabilität der partnerbezogenen Bindungsvermeidung im Vergleich zur partnerbezogenen Bindungsangst. Grau (1999) hatte den postulierten Stabilitätsunterschied von Angst und Vermeidung in der Bindung an einen konkreten Partner nachweisen können. Im Unterschied zu Grau erfassten wir die Bindungsdimensionen beziehungsspezifisch und nicht zielpersonenspezifisch. Die Vermutung liegt nahe, dass die beziehungsspezifische Bindungsangst aufgrund ihrer Repräsentation auf einer höheren Hierarchie-Ebene im kognitiven Netzwerk in einem geringeren Ausmaß als die zielpersonenspezifische Bindungsangst von den aktuellen Interaktionserfahrungen mit einem konkreten Partner abhängt. Das könnte die Diskrepanz der Befunde sehr gut erklären.

Wir nutzten in unserer Untersuchung die Retest-Reliabilitäten der Persönlichkeitsdimensionen Neurotizismus und Verträglichkeit als Bewertungsstandard zur Beurteilung der Retest-Reliabilitäten der Bindungsdimensionen. Entgegen unseren Erwartungen erweisen sich bei diesem Vergleich die Bindungsdimensionen als ähnlich stabil wie die genannten Persönlichkeitsmerkmale, die inhaltlich nah sind. Da aber die internen Konsistenzen anzeigen, dass die Messung von Verträglichkeit

$(=.65$ bis $=.71)$ nicht so reliabel ist, wie die Messung der Bindungsvermeidung ( $=.82$ bis $=.88$ ), ermöglicht der hier durchgeführte Vergleich keine ab- 
schließende Beurteilung. Die niedrige Retest-Reliabilität von Verträglichkeit $\left(\mathrm{r}_{\mathrm{tt}}=\right.$ .67) kann möglicherweise auch auf die relativ geringe interne Konsistenz dieser Skala zurückgeführt werden (Lienert/Raatz 1994: 181).

Was die Frage der relativen Stabilität von partnerbezogener Bindungsangst und Neurotizismus angeht, betrachten wir das vorliegende Ergebnis nur als vorläufig. In größeren Stichproben könnte durchaus ein signifikanter Unterschied zwischen den Retest-Reliabilitäten von Bindungsangst und Neurotizismus festgestellt werden, der aufgrund der kleinen Stichprobe $(N=57)$ hier nicht aufgedeckt werden konnte.

Dass der relativ niedrige Retest-Koeffizient von $r_{t t}=.60$ der partnerbezogenen Bindungsangst auf ihre Veränderbarkeit hinweist (und nicht nur auf Fehlervarianz), legt der gefundene Einfluss von Beziehungsveränderungen auf die Bindungsangst nahe. So zeigt sich ein Zusammenhang zwischen wahrgenommenen Beziehungsveränderungen und Bindungsangst, wenn die Bewertung der Beziehungsveränderungen einbezogen wird. Bei positiv bewerteten Beziehungsveränderungen nimmt die partnerbezogene Bindungsangst ab, während sie bei negativ eingestuften Veränderungen signifikant zunimmt. Davila, Karney und Bradbury (1999) fanden, dass die Sicherheit der Bindung bei Neuverheirateten über einen Zeitraum von zwei Jahren zunimmt. Unser Ergebnis zeigt, dass auch schon geringfügige positive Beziehungsveränderungen zu einer Abnahme der Bindungsangst und damit auch indirekt zu einer höheren Sicherheit der partnerbezogenen Bindung führen. Die Zunahme der partnerbezogenen Bindungsangst im Kontext negativ bewerteter Veränderungen im Beziehungsleben steht im Einklang mit der Untersuchung von Ruvolo, Fabin und Ruvolo (2001), die fanden, dass bei einer Trennung vom Partner bei Studentinnen die Sicherheit der Bindung abnimmt.

Neben den schon von Bowlby beschriebenen langfristigen Veränderungen in den Bindungsrepräsentationen, die eine Adaption an neue interpersonell relevante und deutlich veränderte Lebensumstände darstellen (Collins/Read 1994), werden von Davila und Cobb (2003) auch Bindungsveränderungen angenommen, die sich relativ kurzfristig zeigen. Diese basieren auf dem instabilen Modell vom Selbst und den Anderen. Auch wenn sich im direkten Vergleich keine Unterschiede in der zeitlichen Stabilität der beiden Bindungsdimensionen ergeben, wird die Bindungsvermeidung hier nicht durch Beziehungsveränderungen beeinflusst.

Für die praktische Anwendung kann daraus abgeleitet werden, dass es besonders bei einer ängstlichen Bindungsorientierung günstig ist, in der Partnerschaft positive Beziehungsereignisse zu erleben. Denn die Ergebnisse haben gezeigt, dass sich positive Beziehungsveränderungen in Richtung einer Verringerung der Bindungsängstlichkeit auswirken. Dieses Potenzial einer positiven Beziehungsentwicklung kann eingesetzt werden, um die Unsicherheit in der Bindung abzubauen. Es ist auch darauf hinzuweisen, dass die Beziehungsveränderungen, die zu einer Verringerung der Bindungsangst führten, relativ geringfügig waren. Daher kann angenommen werden, dass sich solche Veränderungen auch in dem täglichen Erleben gezielt implementieren lassen, wenn die Partner das anstreben.

Obwohl wir ganz bewusst Studierende in den ersten Semestern untersuchten, da wir vermuteten, dass bei ihnen Beziehungsveränderungen häufig stattfinden, hatten nur 42 Personen Beziehungsveränderungen angegeben, so dass die Aussage- 
kraft der Befunde eingeschränkt ist. In zukünftigen Untersuchungen wäre es neben dem Ziel, eine größere Stichprobe über einen längeren Zeitraum zu untersuchen, wünschenswert, die Auswirkungen von Beziehungsveränderungen prospektiv zu erfassen, indem beispielsweise Personen vor und nach der Heirat befragt werden.

Wiederholte Messungen haben den Vorteil, dass Längsschnittanalysen durchgeführt werden können, die auf kausale Zusammenhänge verweisen. Ein Nachteil liegt darin, dass über die Messzeitpunkte Teilnehmer der Ausgangsstichprobe verloren gehen. Ein methodisches Problem der vorliegenden Studie ist die Reduktion der Stichprobe. Diese scheint vor allem auf das Studium zurückzuführen zu sein und nicht in Zusammenhang mit den untersuchten Konstrukten zu stehen.

Studiumsbedingt gibt es in unserer Stichprobe einen Überhang weiblicher Probanden. Diese unterscheiden sich jedoch im Ausmaß von Bindungsangst und Bindungsvermeidung zum ersten Messzeitpunkt nicht von den männlichen Befragten $(\mathrm{F}(2,118)=1.384$, n.s. $)$, auch nicht in der Häufigkeit von Lebensveränderungen allgemein $\left(\mathrm{Chi}^{2}(\mathrm{df}=1)=.985\right.$, n.s. $)$ noch in den Veränderungen im Beziehungsleben $\left(\mathrm{Chi}^{2}(\mathrm{df}=1)=.886\right.$, n.s. $)$. Zudem fehlen theoretische Hinweise, die geschlechtsabhängige Unterschiede in der Stabilität der Bindungsdimensionen sowie Unterschiede in der Wirkung von Lebensveränderungen nahe legen würden. Lopez und Gormley (2002) finden in ihrer Längsschnittstudie mit 207 Studierenden keinen Geschlechtseffekt für die absolute Anzahl von Lebensveränderungen und deren Einfluss auf das Wohlbefinden oder den Umgang mit Stress und intrapsychischen Konflikten. Auch der Zusammenhang zwischen Bindungsstiländerung und dem Erleben von Distress (z.B. depressive Verstimmung, persönliche Probleme) wird nicht vom Geschlecht moderiert. Demzufolge dürfte der Überhang von Frauen unter den Befragten die Ergebnisse nicht grundsätzlich beeinflussen. Die Befragten waren mit durchschnittlich 26 Jahren insgesamt verhältnismäßig jung. Dennoch verfügten sie bereits über Beziehungserfahrungen. Über zwei Drittel lebten in einer festen Beziehung oder waren sogar verheiratet, fast die Hälfte hatte bereits Erfahrung mit mehr als zwei längeren Partnerschaften (mindestens sechs Monate) und ebenso vielen kürzeren Partnerschaften (weniger als sechs Monate). Anzunehmen ist, dass Veränderungen im Beziehungsleben mit dem Alter eher ab als zunehmen und die partnerschaftliche Bindung eher stabiler wird. Wir haben bewusst eine Stichprobe ausgewählt, bei der noch mit verhältnismäßig vielen Lebens- und Beziehungsveränderungen zu rechnen ist, bei der also etwaige Einflüsse am ehesten nachweisbar sein sollten. Inwieweit gravierende Beziehungsveränderungen wie eine Scheidung oder der Tod des Lebenspartners nach vielen Jahren einer festen und stabilen Partnerschaft die Bindung auch noch in höherem Alter gravierend beeinflussen, bleibt zu prüfen. Theoretisch sollte ein ähnliches, wenngleich möglicherweise stärker ausgeprägtes Ergebnismuster zu erwarten sein. $\mathrm{Zu}$ bedenken bleibt, dass die verminderte Stichprobengröße zu statistischen Problemen bei der Feststellung von signifikanten Ergebnissen führte, die die Power des Tests betreffen.

Insgesamt zeigen unsere Ergebnisse, dass die partnerbezogene Bindungsangst schon durch geringfügige partnerbezogene Interaktionserfahrungen in kongruenter Weise beeinflusst wird: Positive Erfahrungen wirken sich günstig aus, negative ungünstig. Die partnerbezogene Bindungsvermeidung ist weniger durch partnerbe- 
zogene Interaktionserfahrungen beeinflussbar, was dem ursprünglichen Konzept der frühkindlichen Bindung von Bowlby (1969/1986) entspricht, nach dem die Bindung stabil ist und nur durch gravierende Erfahrungen beeinflusst werden kann. Zudem konnten wir nachweisen, dass der Einfluss des Neurotizismus, der in der eigenen Nervosität und Stressempfindlichkeit zum Ausdruck kommt, auf die partnerbezogene Bindungsangst stärker ist als umgekehrt. In weiteren Untersuchungen sollten Bindungsangst und Bindungsvermeidung sowohl hinsichtlich der Stabilität als auch hinsichtlich der Generalität differenziert betrachtet werden.

\section{Literatur}

Ainsworth, M. D. S./Blehar, M. C./Waters, E./Wall, S. (1978). Patterns of attachment. A psychological study of the strange situation. Hillsdale: Erlbaum.

Asendorpf , J. B. (1999). Psychologie der Persönlichkeit. Berlin: Springer

Asendorpf, J. B./Banse, R./Wilpers, S./Neyer, F. J. (1997). Beziehungsspezifische Bindungsskalen für Erwachsene und ihre Validierung durch Netzwerk- und Tagebuchverfahren. Diagnostica, 43, S. 289-313.

Asendorpf, J. B./Wilpers, S. (1998). Personality effects on social relationships. Journal of Personality and Social Psychology, 74, p. 1531-1544.

Baldwin, M. W./Fehr, B. (1995). On the instability of attachment style ratings. Personal Relationships, 2, p. 247-261.

Baldwin, M. W./Keelan, J. P. R./Fehr, B./Enns, V./Koh-Rangarajoo, E. (1996). Socialcognitive conceptualization of attachment working models: Availability and accessibility effects. Journal of Personality and Social Psychology, 71, p. 94-109.

Bartholomew, K. (1990). Avoidance of intimacy: an attachment perspective. Journal of Social and Personal Relationships, 7, p. 147-178.

Barholomew, K./Horrowitz, L. M. (1991). Attachment styles among young adults. A test of a four-category model. Journal of Personality and Social Psychology, 61, p. 226-244.

Bierhoff, H.-W. (2000). Sozialpsychologie. Stuttgart: Kohlhammer.

Borkenau, P./Ostendorf, F. (1993). NEO-Fünf-Faktoren Inventar (NEO-FFI). Göttingen: Hogrefe.

Bortz, J. (1993). Statistik für Sozialwissenschaftler. Berlin: Springer.

Bowlby, J. (1969/1986). Bindung. Frankfurt: Fischer.

Brennan, K. A./Clark, C. L./Shaver, B. R. (1998). Self report measurement of adult attachment. An integrative overview. In: J. A. Simpson/S. Rholes (Eds.), Attachment theory and close relationships (pp. 46-76). New York: Guilford Press.

Bretherton, I./Munholland, K. A. (1999). Internal working models in attachment relationships. A construct revisited. In: J. Cassidy/P. R. Shaver (Eds.), Handbook of attachment: Theory, research, and clinical application (pp. 89-111). New York: Guilford Press.

Collins, N. L./Read, S. J. (1990). Adult attachment, working models, and relationship quality in dating couples. Journal of Personality and Social Psychology, 58, p. 644-663.

Collins, N. L./Read, S. J. (1994). Cognitive representations of attachment: The structure and function of working models. In: K. Bartholomew/D. Pearlman (Eds.), Advances in personal relationships (Vol. 5, pp. 53-90). London: Jessica Kingsley.

Costa, P. T. Jr./McCrae, R. R. (1985). Revised NEO Personality Inventory (NEO PI-R): Professional manual. Odessa, FL: Psychological Assessment Resources.

Cozzarelli, C./Hoekstra, S. J./Bylsma, W. H. (2000). General versus specific mental models of attachment: Are they associated with different outcomes? Personality and Social Psychology Bulletin, 26, p. 605-618. 
Cozzarelli, C./Karafa, J. A./Collins, N. L./Tagler, M. J. (2003). Stability and change in adult attachment styles: Associations with personal vulnerabilities, life events, and global construals of self and others. Journal of Social and Clinical Psychology, 22, p. 315-346.

Davila, J./Cobb, R. J. (2003). Predicting change in self-reported and interviewer-assessed adult attachment: Tests of individual difference and life stress models of attachment change. Personality and Social Psychology Bulletin, 29, p. 859-870.

Davila, J./Karney, B. R./Bradbury, T. N. (1999). Attachment change processes in the early years of marriage. Journal of Personality and Social Psychology, 76, p. 783-802.

Feeney, J. A. (1999). Adult romantic attachment and couple relationships. In: J. Cassidy/P. R. Shaver (Eds.), Handbook of attachment: Theory, research, and clinical applications (pp. 355-377). New York: Guilford Press.

Fraley, R. C./Waller, N. G. (1998). Adult attachment patterns: A test of typological model. In: J. A. Simpson/W. S. Rholes (Eds.), Attachment theory and close relationships (pp. 77-114). New York: Guilford Press.

Grau, I. (1994). Entwicklung und Validierung eines Inventars zur Erfassung von Bindungsstilen in Paarbeziehungen. Unveröffentlichte Dissertation: Philipps-Universität Marburg.

Grau, I. (1999). Skalen zur Erfassung von Bindungsrepräsentationen in Paarbeziehungen. Zeitschrift für Differentielle und Diagnostische Psychologie, 20, S. 142-152.

Hazan, C./Shaver, P. (1987). Romantic love conceptualized as an attachment process. Journal of Personality and Social Psychology, 52, p. 511-524.

Hazan, C./Zeifman, D. (1999). Pair bonds as attachments. Evaluating the evidence. In: J. Cassidy/P. R. Shaver (Eds.). Handbook of Attachment: Theory, research, and clinical applications (pp. 336-354). New York: Guilford Press.

Heublein, U./Schmelzer, R./Sommer, D. (2005). Studienabbruchstudie 2005. Kurzinformation des Hochschul-Informations-Systems, A1/2005.

Karney, B. R./Bradbury, T. N. (1995). The longitudinal course of marital quality and stability: A review of theory, method and research. Psychological Bulletin, 118, p. 3-34.

Kenny, D. A. (1975). Cross-lagged panel correlation: A test for spuriousness. Psychological Bulletin, 82, p. 887-903.

Kirkpatrick, L. A./Hazan, C. (1994). Attachment styles and close relationships: A four-year prospective study. Personal Relationships, 1, p. 123-142.

Küpper, B. (2002). Sind Singles anders? Göttingen: Hogrefe.

Lienert, G./Raatz, U. (1994). Testaufbau und Testanalyse. Weinheim: Beltz.

Lopez, F. G./Gormley, B. (2002). Stability and change in adult attachment style over the first-year college transition: Relations to self-confidence, coping and distress pattern. Journal of Counseling Psychology, 49, p. 355-364.

Maccoby, E. E. (1980). Social development. New York: Harcourt Brace.

Mikula, G./Leitner, A. (1998). Partnerschaftsbezogene Bindungsstile und Verhaltenserwartungen an Liebespartner, Freunde und Kollegen. Zeitschrift für Sozialpsychologie, 29, S. 213-223.

Neyer, F. J. (2003). Persönlichkeit und Partnerschaft. In: H. W. Bierhoff/I. Grau (Hrsg.), Sozialpsychologie der Partnerschaft (S. 165-189). Berlin: Springer.

Raghunathan, T. E./Rosenthal, R./Rubin, B. (1996). Comparing corelated but nonoverlapping correlations. Psychological Methods, 1, p. 178-183.

Rohmann, E. (2000). Gerechtigkeitserleben und Erwartungserfüllung in Partnerschaften. Frankfurt: Lang.

Rohmann, E./Bierhoff, H.W./Schmohr, M./Ambrosy, B. (1998). Verlauf des partnerbezogenen Bindungsstils über die Zeit. Posterbeitrag 41. Kongress der Deutschen Gesellschaft für Psychologie, Dresden.

Ross, L. R./Spinner, B. (2001). General and specific attachment representations in adulthood: Is there a relationship? Journal of Social and Personal Relationships, 18, p. 747766. 
Rudinger, G./Bierhoff, H. W. (1980). Quasi-Experimentelle Versuchspläne für die Marktund Kommunikationspsychologie. In: K. D. Hartmann/K. Koeppler (Hrsg), Fortschritte der Marktpsychologie, Bd. 2 (S. 135-163). Fachbuchhandlung für Psychologie GmbH. Frankfurt.

Ruvolo, A. P./Fabin, L. A./Ruvolo, C. M. (2001). Relationship experiences and change in attachment characteristics of young adults: The role of relationship breakups and conflict avoidance. Personal Relationships, 8, p. 265-281.

Scharfe, E./Bartholomew, K. (1994). Reliability and stability of adult attachment patterns. Personal Relationships, 1, p. 23-43.

Schmohr, M. (2003). Was macht Partnerschaften glücklich und stabil? Ein Test des Investitionsmodels unter Berücksichtigung der Bindungsstile. Hamburg: Verlag Dr. Kovac.

Shaver, P. R./Brennan, K. A. (1992). Attachment styles and the "Big Five" personality traits: Their connections with each other and with romantic relationship outcomes. Personality and Social Psychology Bulletin, 18, p. 536-545.

Shaver, P. R./Clark, C. L. (1996). Forms of adult romantic attachment and their cognitive and emotional underpinnings. In: G.G. Noam/K. W. Fischer (Eds.). Development and vulnerability in close relationships (S. 29-58). New York: Lawrence Erlbaum.

Shaver, P. R./Collins, N./Clark, C. L. (1996). Attachment styles and internal working models of self and relationship partners. In: G. J. O. Fletcher/J. Fitness (Eds.). Knowledge structures in close relationships: A social psychological approach (S. 25-61). Hillsdale: Lawrence Erlbaum.

Shaver, P. R./Mikulincer, M. (2002a). Attachment-related psychodynamics. Attachment and Human Development, 4, p. 133-161.

Shaver, P. R./Mikulincer, M. (2002b). Dialogue on adult attachment: Diversity and integration. Attachment and Human Development, 4, p. 243-257.

Sprecher, S. (1986). The relation between inequity and emotions in close relationships. Social Psychology Quarterly, 49, p. 309-321.

Zimmermann, P. (1999). Structure and functions of internal working models of attachment and their role for emotion regulation. Attachment and Human Development, 1, p. 291306.

Eingereicht am: 14.12.2004

Akzeptiert am: 30.04.2005

\title{
Anschrift der Autorinnen
}

\author{
Dr. Elke Rohmann \\ Ruhr-Universität Bochum \\ Fakultät für Psychologie \\ Sozialpsychologie \\ Universitätsstr. 150 \\ D-44780 Bochum
}

Email: elke.rohmann@rub.de

Dr. Beate Küpper

Institut für interdisziplinäre Konflikt- und Gewaltforschung 
Universität Bielefeld

Universitätsstr. 25

33615 Bielefeld

Email: beate.kuepper@uni-bielefeld.de 